\section{Ingrid Weber}

zum 70. Geburtstag. Ingrid Weber ist Vorsitzende Richterin am Landesarbeitsgericht Berlin i.R. und leidenschaftliche Arbeitsrechtlerin auch über ihre berufliche Tätigkeit hinaus. Für die Gleichstellung der Geschlechter und diskriminierungsfreie Arbeits- und Lebensbedingungen hat sie viel erreicht.

Ein besonderes Anliegen sind ihr die Gleichstellungsgesetze allen voran in Berlin. Das Berliner Frauenfördergesetz (später umbenannt in LGG Berlin) trat vor 15 Jahren in Kraft. Die damals zuständige Senatorin Anne Klein hatte im Vorfeld eine Expertinnenrunde einberufen, deren Mitglied Ingrid Weber war. Berlin hatte mit der Schaffung des Gesetzes eine Vorreiterrolle, die Widerstände waren beträchtlich. Ingrid Weber moderierte viele Veranstaltungen zum LGG, um für die gesetzlichen Regelungen zu werben. Zehn Jahre später war sie als Sachverständige und Moderatorin ebenfalls beteiligt, als die Rechtsverordnung zu formulieren war, mit der die Koppelung öffentlicher Auftragsvergabe mit Frauenförderung näher ausgestaltet wurde.

Frauenpolitisch ebenfalls sehr wichtig ist ihr die Gleichstellung der Geschlechter in der Privatwirtschaft. Dr. Bergmann berief 1999 als zuständige Bundesministerin eine Expertenrunde mit Vertretern und Vertreterinnen aus Wissenschaft und Praxis, Unternehmen und Gewerkschaften ein, der Ingrid Weber als frauenpolitisch engagierte Richterin angehörte. Es ging darum, Rahmenbedingungen in Hinblick auf die Akzeptanz eines entsprechenden Gesetzes abzuklären. 2000 beauftragte die Ministerin eine sechsköpfige Expertinnengruppe unter Führung von Prof. Dr. Heide Pfarr mit der Formulierung eines Gesetzentwurfs, der 2001 mit ihrer tatkräftigen Unterstützung vorgelegt wurde und auch heute noch Ausgangspunkt für die Diskussion um ein Gesetz zur Gleichstellung der Geschlechter in der Privatwirtschaft ist.

Ingrid Weber engagiert sich seit 1986 im Deutschen Juristinnenbund und war in mehreren Kommissionen und Arbeitsstäben Mitglied (Arbeitsrecht und moderne Technologien, Gleichstellungskommission, Arbeits- und Sozialrecht, Deutsch-deutsche Rechtsbeziehungen). Von 2001 bis 2005 leitete sie die Kommission Arbeits-, Gleichstellungs- und Wirtschaftsrecht, war in dieser Funktion auch Mitglied des Bundesvorstandes und verantwortlich für zahlreiche wichtige Stellungnahmen des djb. Alle Stellungnahmen entstanden in echter Teamarbeit, Ingrid Weber leitete die Kommission unprätentiös und förderte die besonderen Stärken jedes einzelnen Kommissionsmitgliedes. Nicht nur innerhalb der Kommission sondern auch im Vorstand hatte sie hohe Integrationskraft und dies auch bei kniffligen Themen und manchmal schwierigen Abstimmungsprozessen.

Ingrid Weber gehört zu den Frauen im djb, die andere anspornen und begeistern können. Sie nimmt auch und gerade jüngere und unbekanntere Mitglieder des djb ernst und integriert sie auf heitere Weise in die gemeinsame Arbeit. Ihre Gastfreundlichkeit ist legendär und ihr Rat nach wie vor willkommen. Ich persönlich habe unglaublich viel von ihr gelernt. (Christine Fuchsloch)

\title{
Women Power in Brussels - how and why do women lobby differently?
}

\section{Podiumsdiskussion am 20. September 2010 in der Nordrhein-Westfälischen Landesvertretung in Brüssel}

\section{Dr. Claudia Schöler}

Mitglied im Vorstand der Regionalgruppe Brüssel des djb; VDMA European Office, Brüssel

„Women Power in Brussels - how and why do women lobby differently? “ - unter diesem Motto lud die Regionalgruppe Brüssel im djb zusammen mit dem niederländischen Frauennetzwerk „Brussels NV“ am 20. September 2010 zu einer Podiumsdiskussion in die Nordrhein-Westfälische Landesvertretung in der EU-Hauptstadt. Zwei prominente Brüsseler Interessenvertreterinnen gaben Einblick in ihre politische Arbeit: Constanze Picking, Direktorin der EU-Unternehmensrepräsentanz von Daimler, und Truus Huisman, Leiterin des Public Affairs-Büros von Unilever in Brüssel. Carla Joosten, Korrespondentin des niederländischen Politik-Magazins „Elsevier“ führte durch die Diskussion.
Gesprächsstoff gab es reichlich - angesichts der hohen Sprosse, die beide Damen auf der Karriereleiter erklommen haben, lagen Fragen zum persönlichen beruflichen Werdegang und der Vereinbarkeit von Beruf und Familie genauso auf der Hand wie Fragen zur Kunst der politischen Interessenvertretung in der EU-Hauptstadt, in der sich über 15.000 Lobbyisten tummeln. Wie haben es beide Frauen in der „Männerwelt“ ihrer Unternehmen „nach oben“ geschafft? Brauchen wir die Frauenquote und gibt es die viel gescholtene „gläserne Decke“ (letztere, das wurde schnell klar, war eine rhetorische Frage!)? Wie hat sich die politische Interessenvertretung im Laufe der Jahre verändert, welche Rolle spielt die Transparenzinitiative der EU-Institutionen und inwieweit kann die Industrie von Kampagnen der Nichtregierungsorganisationen lernen? Auch die Frage nach Erfolgsgeschichten und weniger erfolgreichem Lobbying war erlaubt. Offene Worte, interessante Einblicke 


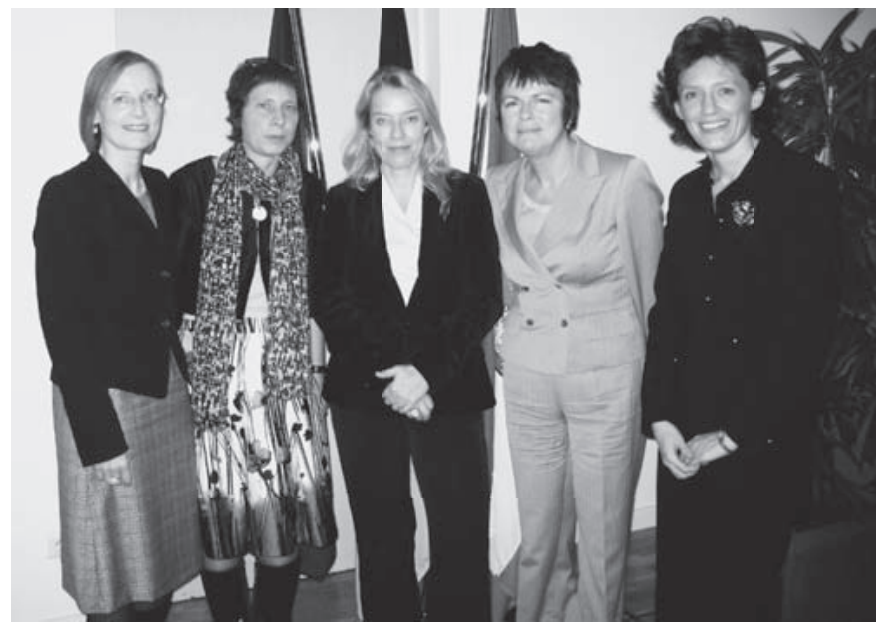

und auch hilfreiche Ratschläge für Kolleginnen führten zu anregenden Diskussionen der mehr als 60 Teilnehmerinnen, die im Anschluss noch beim Empfang in der Landesvertretung Nordrhein-Westfalen vertieft werden konnten.

\section{Aktuelle Pressemitteilungen und Stellungnahmen}

\section{Pressemitteilungen}

10-27 40 Prozent Frauen in die Aufsichtsräte - djb begrüßt Gesetzentwurf Bündnis 90/Die Grünen. (Oktober 2010)

10-26 Kabinettsbeschluss zum GKV-Finanzierungsgesetz: djb sieht Frauen als Verliererinnen der Reform. (September 2010)

10-25 Minijobs wirken der gleichstellungspolitisch notwendigen eigenständigen Existenzsicherung von Frauen entgegen. (September 2010)

10-24 Juristinnen präsentieren „differenzierte Widerspruchslösung “: Neue Regelung der elterlichen Sorge „nichtehelicher“ Väter. (September 2010)

10-23 Tarifverhandlungen im öffentlichen Dienst: Hoffnung auf ein Ende der diskriminierenden Arbeitsbewertung. djb begrüßt Vorhaben einer diskriminierungsfreien Entgeltordnung im Bereich des TVöD. (August 2010)

10-22 Gemeinsame Sorge nichtehelicher Eltern: Justizministerin vollzieht Kehrtwende zu Lasten unverheirateter Mütter. (August 2010)

Endlich die tatsächliche erbrechtliche Gleichstellung nichtehelicher Kinder! (August 2010)

10-20 Deutsche Vorbehalte zur UN-Kinderrechtskonvention zurückgenommen. (Juli 2010)

10-19 Dr. Christine Fuchsloch erste Präsidentin des LSG Schleswig-Holstein. ( Juni 2010)

10-18 Frauen und (Lego-)Technik ... djb-Aktion „Aktionärinnen fordern Gleichberechtigung “. (Juni 2010)

10-17 Aufsichtsräte müssen Corporate-Governance-Vokabeln. djb-Aktion „Aktionärinnen fordern Gleichberechtigung!“ (Juni 2010)

10-16 (K)ein Ende in Sicht? djb-Aktion „Aktionärinnen fordern Gleichberechtigung!“ (Juni 2010)

10-15 60 mal gefragt - djb-Aktion „Aktionärinnen fordern Gleichberechtigung“. (Mai 2010)
10-14 BRAK-Präsident Filges ernennt Dr. h.c. Renate Jaeger zur Schlichterin der Rechtsanwaltschaft. (April 2010)

10-13 Härtefallregelung im Hartz IV-Bezug: Gesetzgebungsvorhaben lässt die gebotene Transparenz vermissen. (April 2010)

10-12 Sag mir, wo die Frauen sind ... djb-Aktion „Hauptversammlungen: Aktionärinnen fordern ein!“ geht weiter (Daimler). (April 2010)

\section{Stellungnahmen}

10-12 Stellungnahme zu den Vorlagebeschlüssen des Bundessozialgerichts, $1 \mathrm{BvL} 2 / 10,1 \mathrm{BvL} 3 / 10$ und $1 \mathrm{BvL}$ 4/10. (August 2010)

10-11 Stellungnahme zum Vorlagebeschluss des Sozialgerichts München vom 10. 12. 2007 - S 29 EG 59/07. (August 2010)

10-10 Stellungnahme zu der Verfassungsbeschwerde $1 \mathrm{BvR}$ 918/10. (Juli 2010)

10-09 Stellungnahme zum Entwurf eines Beitrages zum Hausbegleitgesetz 2011 (Referentenentwurf) - Änderungen des BEEG (Stand des Entwurfs: 9.7.2010) anlässlich der Verbändebeteiligung gemäß $\mathbb{4} 4$ Abs. 3 GGO am 28. Juli 2010. (Juli 2010)

Stellungnahme zum Entwurf für ein „Gesetz zur Stärkung der Pressefreiheit im Straf- und Strafprozessrecht“ (PrStG). (Juni 2010)

10-07 Stellungnahme zum 9. Gesetz zur Änderung des Landesgleichstellungsgesetzes. Entwurf der Senatsverwaltung für Wirtschaft, Technik und Frauen IV A/ IV A 11. (Mai 2010)

10-06 Stellungnahme zum Zweiten Gesetz zur erbrechtlichen Gleichstellung nichtehelicher Kinder (Referentenentwurf des BMJ, Stand 1.12.2009). (April 2010) 